\title{
Capacity Bounds for an Ultra-Wideband Channel Model
}

\author{
Erdal Arikan ${ }^{1}$ \\ Dept. of Electrical-Electronics Engineering \\ Bilkent University \\ Ankara, TR-06800, Turkey \\ e-mail: arikan@ee.bilkent.edu.tr
}

\begin{abstract}
There is an ongoing effort by the IEEE 802.15.3a subcommittee to reach a UWB personal area network standard. We estimate the achievable rates for such networks using a channel model specified by the same group. The analysis of this channel model is of interest in light of recent informationtheoretic work on multipath fading channels which show that in order to take full advantage of such channels' capacity the transmitted signals have to be "peaky" in a certain sense. The immense bandwidth of the UWB channel also suggests at first that peaky signals should be used. However, unlike the many other wireless systems where the transmitter energy is limited, in the UWB channel only the power spectral density of the transmitted signal is constrained. As a result, the signal power can grow in proportion to the utilized bandwidth and peaky signals are not needed.
\end{abstract}

\section{INTRODUCTION}

Initiated by FCC's release of the 3.1-10.6 GHz band for unlicensed operation under certain restrictions [1], there is an ongoing standardization effort by IEEE subcommittee 802.15.3a aimed at creating a UWB physical layer for wireless personal area networks. The question of interest in this paper is to estimate the capacity of the UWB channel. We begin with an initial estimate of the main operating parameters for such systems.

The FCC ruling stipulates that the emitted power spectral density of signals be at most $-41.3 \mathrm{dBm} / \mathrm{MHz}$ within the above frequency range. First, we note that even if the transmitter utilizes the entire permissible bandwidth of $7.5 \mathrm{GHz}$, the total radiated power is $-2.55 \mathrm{dBm}$, which is feasible even for battery operated devices. So, the UWB system is not limited by available transmitter power.

Consider a UWB system employing signals of RF bandwidth $W$ and duration $T$. The effective signal energy at the receiver can be expressed as $\mathcal{E}=\eta W T L$ where $\eta=-41$ $\mathrm{dBm} / \mathrm{MHz}$ and $L$ is the loss factor from transmitter to receiver. The received energy per degree of freedom equals $\mathcal{E}_{s}=\mathcal{E} / W T=\eta L$. We may write the loss $L$ as consisting of two parts $L=L_{1} L_{2}$ where $L_{1}$ is due to radio-wave propagation, and $L_{2}$ due to receiver non-idealities. Under free-space conditions, $L_{1}$ may be estimated by the Friis formula $L_{1}=\left(\frac{\lambda_{c}}{4 \pi d}\right)^{2}$ where we assume the receiver and transmitter antennas are isotropic and separated by a distance $d$, and take $\lambda_{c}$ as the wavelength corresponding to the frequency $f_{c}=5092 \mathrm{MHz}$. There is no fundamental reason for using this particular value for $f_{c}$ other than being able to compare our results with those in a proposed standard [3]. The use of a

\footnotetext{
${ }^{1}$ This work was supported by European Union FP-6 Project NEWCOM, Contract IST NoE 507325.
}

single frequency to estimate the propagation loss over a wide range of frequencies is only an approximation; we refer to [2] for a discussion of the error in this approximation. We take $L_{2}=10 \mathrm{~dB}$ to account for receiver noise and other losses.

Assuming the dominant noise in the system is white Gaussian with one-sided power-spectral density $N_{0}=-114$ $\mathrm{dBm} / \mathrm{MHz}$, we obtain the SNR figures $\mathcal{E}_{s} / N_{0}=\eta L / N_{0}$ listed in the following table. Also given in the table is the AWGN capacity $C=W \log \left(1+\frac{\varepsilon_{x}}{N_{0}}\right)$ for an RF bandwidth of $W=500$ $\mathrm{MHz}$, which is the minimum allowed bandwidth for a UWB system. The AWGN capacity may serve as a rough estimate of what range of rates may be expected in the UWB channel. Note that the AWGN capacity scales linearly with $W$ since the signal power is allowed to grow linearly with $W$.

\begin{tabular}{|l|c|c|c|c|}
\hline$d(\mathrm{~m})$ & 1 & 4 & 10 & 20 \\
\hline $\mathcal{E}_{s} / N_{0}(\mathrm{~dB})$ & 16.12 & 4.08 & -3.88 & -9.90 \\
\hline$C_{\text {AWGN }}$ (Mbps) & 2695.0 & 915.6 & 247.5 & 70.3 \\
\hline
\end{tabular}

Table 1: Signal-to-noise ratio and capacity estimates for the UWB channel with $W=500 \mathrm{MHz}$ RF bandwidth.

\section{IEEE UWB CHANNEL MODEL}

IEEE 802.15.3a group published a channel model for UWB communications [4]. The channel is modeled as a linear system with an impulse response

$$
h(t)=\beta \sum_{\ell} \sum_{i} \alpha_{i, \ell} \delta\left(t-T_{\ell}-\tau_{i, \ell}\right)
$$

where $\alpha_{i, \ell}$ is the path gain for the $i$ th "ray" in the $\ell$ th "cluster." The number of clusters and rays within each cluster are random variables, as well as the path gains $\alpha_{i \ell} . T_{\ell}$ is the random delay for the $\ell$ th cluster and $\tau_{i, \ell}$ is the excess delay for the $i$ th ray in the $\ell$ th cluster. The term $\beta$ represents a common "shadowing" gain, which is independent of the $\left\{\alpha_{i, \ell}\right\}$. All gain terms are real-valued random variables whose exact distributions can be found in [4]. For our purposes, it is only important to note that $\left\{\alpha_{i, \ell}\right\}$ are specified as uncorrelated random variables with symmetric distributions around the origin.

In [4] four sets of parameters are defined, denoted as CM1CM4, modeling various UWB environments. Some of the properties of these channel models are given in Table 2 . The values in the table are computed after time-discretization of the fading coefficients $\left\{\beta \alpha_{i, \ell}\right\}$ using a sampling period of $T_{s}=167 \mathrm{ps}$. In this table, NP $10 \mathrm{~dB}$ stands for the number of paths whose intensity is within $10 \mathrm{~dB}$ of the intensity of the path with maximum energy. Likewise, NP (85\%) stands for the number of paths that capture $85 \%$ of the channel energy. 
Channel energy is defined as the sum of the squared absolute values of the path gains. All quantities in the table represent averages over 100 sample realizations for each channel model. For exact details, we refer to [4].

\begin{tabular}{|c|c|c|c|c|}
\hline Model characteristics & CM1 & CM2 & CM3 & CM4 \\
\hline Coherence time $\overline{\overline{T_{c}(\mu \mathrm{s})}}$ & 200 & 200 & 200 & 200 \\
\hline RMS delay spread (ns) & 5.28 & 8.03 & 14.28 & 25 \\
\hline$N P_{10 \mathrm{~dB}}$ & 13.3 & 18.2 & 25.3 & 41.4 \\
\hline$N P(85 \%)$ & 21.4 & 37.2 & 62.7 & 122.8 \\
\hline Channel energy (dB) & -0.5 & 0.1 & 0.2 & 0.1 \\
\hline
\end{tabular}

Table 2: IEEE UWB channel model characteristics.

\section{Baseband Channel Model}

A UWB communication system is a bandpass system around some center frequency $f_{c}$ with a bandwidth $W$ contained in the range 3.1 to $10.6 \mathrm{GHz}$. As usual, we will antalyze such a system after signals are translated to the baseband and time-discretized by sampling at the Nyquist rate $T_{s}=1 / W$.

The equivalent complex baseband communication system will be modeled as a discrete-time vector channel where the transmitter sends a complex vector $x=\left(x_{0}, \ldots, x_{K-1}\right)^{T}$ and the receiver observes an output vector $y=\left(y_{0}, \ldots, y_{K-1}\right)^{T}$ given by

$$
y_{i}=\sum_{k=0}^{K-1} g_{k} x_{(i-k)}+z_{i}, \quad i=0, \ldots, K-1
$$

where the additive noise vector $z=\left(z_{0}, \ldots, z_{K-1}\right)^{T}$ is complex Gaussian with circularly symmetric (c.s.) independent components $z_{i} \sim C N\left(0, N_{0}\right)$. (A random vector $z$ is said to be c.s. if $e^{j \theta} z$ has the same distribution as $z$ for any real $\theta$.) The baseband channel coefficient vector $g=\left(g_{0}, \ldots, g_{K-1}\right)^{T}$ is related to the original continuous-time channel impulse response by

$$
g_{k}=\sum_{i, \ell:\left\lfloor d_{i, \ell} / T_{n}\right\rfloor=k} \beta \alpha_{i, \ell} e^{-j 2 \pi f_{c} d_{i, \ell}}
$$

where $d_{i, \ell}=T_{\ell}+r_{i, \ell}$. Since $\left\{\alpha_{i, \ell}\right\}$ are zero-mean and uncorrelated, $\left\{g_{k}\right\}$ are also zero-mean and uncorrelated. Thus, the covariance matrix $C_{g} \triangleq E\left(g g^{\dagger}\right)=\operatorname{diag}\left(\widehat{\left|g_{k}\right|^{2}}\right)$. It is important to note that in the 802.15.3a UWB model, the coefficients $\left\{g_{k}\right\}$ are neither Gaussian nor independent.

The DT channel model (2) is a simplified model in that it assumes the channel state vector $g$ stays fixed for a duration of $K$ symbols. We further asssume that an independent sample of $g$ is selected at random for each new block of $K$ symbols. Thus, the final model is a memoryless vector channel. The vector length $K$ is related to the coherence time $T_{c}$ of the underlying channel by $K=T_{c} / T_{s}$ where $T_{s}$ is the sampling period. Typical values are $T_{c}=200 \mu$ s and $500 \leq W \leq 7500$ $\mathrm{MHz}$, yielding $10^{5} \leq K \leq 1.5 \cdot 10^{6}$.

The summation in (2) is a circular convolution operation with index $(i-k)$ taken modulo $K$. We will use the notation $g \circledast x$ to denote the circular convolution. The idea of using circular convolution instead of linear convolution goes back to Hirt and Massey [5], who used it to simplify the analysis of channels with intersymbol interference. This idea has also been exploited in [6], whose results are particularly relevant in the present context. To estimate the error introduced by using circular convolution, note that the maximum path delay $T_{d}$ for the UWB channel is on the order of $100 \mathrm{~ns}$. So, the edge effects distort a fraction $T_{d} / T_{c} \approx 10^{-3}$ of each transmitted block, which is clearly negligible. In fact, one can introduce a cyclic prefix as in OFDM systems at negligible overhead to remove the modeling inaccuracy due to use of circular convolution.

The channel model (2) is given in the time domain. An equivalent channel can be defined in the frequency domain by means of discrete Fourier transform. The DFT of a vector $a=\left(a_{0}, \ldots, a_{K-1}\right)^{T}$ is defined as the vector $A=$ $\left(A_{0}, \ldots, A_{K-1}\right)^{T}$ given by the unitary transformation $A=F a$ with $F_{k i}=\frac{1}{\sqrt{K}} e^{-j 2 \pi k i / K}$. Taking the DFT of the two sides of (2), we obtain the frequency domain channel

$$
Y_{k}=\sqrt{K} G_{k} X_{k}+Z_{k}, \quad k=0, \ldots, K-1
$$

The noise terms $\left\{Z_{k}\right\}$ are i.i.d. $C N\left(0, N_{0}\right)$. The covariance of $G$ is given by $C_{G}=F G_{g} F^{\dagger}$ and has elements

$$
C_{G}(i, k)=\frac{1}{K} \sum_{\ell} \overline{\left|g_{\ell}\right|^{2}} e^{-j 2 \pi \ell(i-k) / K}
$$

The energy in a CT complex baseband signal $x(t)$ of bandwidth $W / 2$ (corresponding to an RF bandwidth of $W$ ) and duration $T_{c}$ is constrained in a UWB system by

$$
\int_{0}^{T_{c}}|x(t)|^{2} d t \leq \eta L W T_{c}=\mathcal{E}_{s} W T_{c}
$$

where the terms are as defined in Sect. I. For the sampled system this constraint translates into

$$
\sum_{i=0}^{K-1}\left|x_{i}\right|^{2} \leq \mathcal{E}_{\mathrm{s}} W T_{\mathrm{c}}=\mathcal{E}_{\mathrm{s}} K
$$

or equivalently

$$
\sum_{k=0}^{K-1}\left|X_{k}\right|^{2} \leq \mathcal{E}_{s} K
$$

Thus, the parameter $\mathcal{E}_{s}$ may be interpreted as signal energy per complex baseband sample.

\section{UPPER BOUNDS ON ACHIEVABLE RATES}

In this section, we give upper bounds on the achievable rate $I(X ; Y)$ for the channel (4) under various assumptions about the distribution of $X$. These upper bounds will clearly apply also to the achievable rate $I(x ; y)$ for the equivalent channel $(2)$ since $I(x ; y)=I(X ; Y)$. Likewise, $I(X ; Y \mid G)=I(x ; y \mid g)$, etc. The basis of the upper bounds in this section is the identity

$$
\begin{aligned}
I(X ; Y) & =I(X G ; Y)-I(G ; Y \mid X) \\
& =I(S ; Y)-I(G ; Y \mid X)
\end{aligned}
$$

where $S=\left(S_{0}, \ldots, S_{K-1}\right)^{T}, S_{k}=\sqrt{K} G_{k} X_{k}$. We will obtain upper bounds on $l(X ; Y)$ by majorizing $I(S ; Y)$ and minorizing $I(G ; Y \mid X)$ under certain additional assumptions.

Note that the covariance of $S$ has the form $C_{S}=K C_{G} \circ C_{X}$ where $o$ is the Hadamard product, i.e., entrywise product, of the two matrices. When $C_{X}=\mathcal{E}_{s} I_{K}$ (equivalently, when 
$C_{x}=\mathcal{E}_{s} I_{K}$ since $\left.C_{x}=F^{\dagger} C_{X} F\right)$, as we will usually assume, we obtain from (5) that

$$
C_{S}=K \mathcal{E}_{s} \operatorname{diag}\left(C_{G}\right)=\mathcal{E}_{s} \overline{\|g\|^{2}} I_{K}
$$

where diag $(A)$ denotes the diagonal matrix obtained by taking the main diagonal of $A$ and $\|g\|$ denotes the Euclidean norm of $g$.

Lemma 1 For $C_{X}=\mathcal{E}_{s} I_{K}$,

$$
I(S ; Y) \leq K \log \left(1+\frac{\mathcal{E}_{s}}{N_{0}} \overline{\|g\|^{2}}\right)
$$

Proof. Since the channel $Y=S+Z$ is additive Gaussian, the maximum of $I(S ; Y)$ subject to the constraint $C_{S}=\mathcal{E}_{s}\|g\|^{2} I_{K}$ is achieved by $S \sim C N\left(0, C_{S}\right)$ and equals the RHS of (11).

Lemma 2 Cutoff rate bound: Without any restrictions on the channel input $X$ and the coefficients $G$,

$$
I(G ; Y \mid X) \geq E_{X}\left\{-\log E_{G, G^{\prime}}\left[e^{-\frac{K}{4 N_{0}}\left\|X \circ\left(G-G^{\prime}\right)\right\|^{2}}\right]\right\}
$$

where $G^{\prime}$ is an independent copy of $G$. Equivalently,

$$
I(g ; y \mid x) \geq E_{x}\left\{-\log E_{g, g^{\prime}}\left[e^{-\frac{1}{4 N_{0}}\left\|x \circledast\left(g-g^{\prime}\right)\right\|^{2}}\right]\right\}
$$

where $g^{\prime}$ is an independent copy of $g$.

Proof. It is well-known that a mutual information term such as $I(G ; Y \mid X)$ is lower-bounded by the corresponding "cutoff rate" term $R_{0}(G ; Y \mid X)$, which is defined as

$$
E_{X}\left\{-\log E_{G, G^{\prime}}\left[\int \sqrt{p(Y \mid G, X) p\left(Y \mid G^{\prime}, X\right)} d Y\right]\right\}
$$

Denoting the integral in the above expression by $D\left(G, G^{\prime} \mid X\right)$, a straightforward integration gives

$$
D\left(G, G^{\prime} \mid X\right)=\exp \left(-\frac{K}{4 N_{0}}\left\|X \circ\left(G-G^{\prime}\right)\right\|^{2}\right)
$$

and completes the proof of (12). Inequality (13) follows' from $I(g ; y \mid x) \geq R_{0}(g ; y \mid x)$ by showing that $D\left(g, g^{\prime} \mid x\right)=$ $\exp \left(-\frac{1}{4 N_{0}}\left\|x \circledast\left(g-g^{\prime}\right)\right\|^{2}\right)$.

The above results can be combined to give the following upper bound on $I(X ; Y)$.

Proposition 1 Suppose the multipath coefficients $\left\{g_{k}\right\}$ of the channel (2) are zero-mean uncorrelated random variables. Suppose the equivalent frequency domain channel (4) has an input $X$ such that $C_{X}=\mathcal{E}_{s} I_{K}$. Then, the achievable rate is bounded as

$$
\begin{aligned}
I(X ; Y) & \leq K \log \left(1+\frac{\mathcal{E}_{s}}{N_{0}} \overline{\|g\|^{2}}\right)- \\
& E_{X}\left\{-\log E_{G, G^{\prime}}\left[e^{-\frac{K}{4 N_{0}}\left\|X \circ\left(G-G^{\prime}\right)\right\|^{2}}\right]\right\}
\end{aligned}
$$

Remark. This result makes minimal assumptions about the distribution of $g$. However, it has an ad-hoc nature due to the mixed use of the cutoff rate and mutual information. Better and more elegant bounds can be obtained if we make further assumptions about the distributions of $g$ and $X$.
Lemma 3 Assume that the vertor of channel coefficients $g$ is complex Gaussian with c.s. independent components. Let the input $X$ to the channel (4) have covariance $C_{X}=\mathcal{E}_{s} I_{K}$ and further assume that $\left|X_{k}\right|=\sqrt{\mathcal{E}_{s}}$ for all $k$. Then,

$$
I(G ; Y \mid X)=\sum_{i=0}^{K-1} \log \left(1+\frac{\mathcal{E}_{s}}{N_{0}} K \overline{\left|g_{i}\right|^{2}}\right)
$$

Remark. QPSK signaling, which is the signaling scheme in a leading proposed UWB standard [3], satisfies the assumptions of this lemma on $X$.

Proof. Given $X$, the situation is equivalent to an additive Gaussian noise channel with a Gaussian input $G$. So,

$$
I(G ; Y \mid X)=E_{X} \log \operatorname{det}\left(I_{K}+\frac{K}{N_{0}} X X^{\dagger} \circ C_{G}\right)
$$

It can be verified easily that if $e=\left(e_{0}, \ldots, e_{K-1}\right)^{T}$ is an eigenvalue of $C_{G}$ with eigenvalue $\lambda$, then $f=\left(f_{0}, \ldots, f_{K-1}\right)^{T}$ with $f_{i}=e_{i} / X_{i}^{*}$ is an eigenvalue of $X X^{\dagger} \circ C_{G}$ with eigenvalue $\mathcal{E}_{s} \lambda$. This is true for any vector $X=\left(X_{0}, \ldots, X_{K-1}\right)^{T}$ whose elements have a constant modulus $\left|X_{i}\right|=\sqrt{\mathcal{E}_{s}}>0$. Thus,

$$
\begin{aligned}
\operatorname{det}\left(I_{K}+\frac{K}{N_{0}} X X^{\dagger} \circ C_{G}\right) & =\operatorname{det}\left(I_{K}+\frac{K \mathcal{E}_{s}}{N_{0}} C_{G}\right) \\
& =\operatorname{det}\left(I_{K}+\frac{K \mathcal{E}_{s}}{N_{0}} F C_{g} F^{\dagger}\right) \\
& =\operatorname{det}\left(I_{K}+\frac{K \mathcal{E}_{s}}{N_{0}} C_{g}\right) \\
& =\prod_{i=1}^{K}\left(1+\frac{K \mathcal{E}_{s}}{N_{0}} \overline{\left|g_{i}\right|^{2}}\right)
\end{aligned}
$$

and the proof is complete. A shorter proof can be given by observing that given $X$, in effect we have the vector channel $\tilde{Y}=G+\tilde{Z}$, where $\tilde{Y}_{k}=Y_{k} /\left(\sqrt{K} X_{k}\right), \tilde{Z}_{k}=Z_{k} /\left(\sqrt{K} X_{k}\right)$, and $\bar{Z} \sim C N\left(0, N_{0} /\left(K \mathcal{E}_{s}\right)\right)$ independent of $X$.

Proposition 2 Suppose the multipath coefficients $\left\{g_{k}\right\}$ are independent c.s. complex Gaussian. Suppose the channel input in the frequency domain $X$ has covariance $C_{X}=\mathcal{E}_{s} I_{K}$ and its elements have a constant modulus, $\left|X_{k}\right|=\sqrt{\mathcal{E}_{s}}$. Then,

$$
I(X ; Y) \leq K \log \left(1+\frac{\mathcal{E}_{s}}{N_{0}} \overline{\|g\|^{2}}\right)-\sum_{i=0}^{K-1} \log \left(1+\frac{\mathcal{E}_{s}}{N_{0}} \overline{K \mid \overline{\left.g_{i}\right|^{2}}}\right)
$$

Remark: A weakened version of $(20)$ is

$$
I(X ; Y) \leq \frac{K^{2} \mathcal{E}_{s}^{2} \log (e)}{2 N_{0}^{2}} \sum_{i=0}^{K-1}\left[\overline{\left|g_{i}\right|^{2}}\right]^{2}
$$

which is obtained by using the inequalities $\ln (1+a) \leq a$ and $\ln (1+a) \geq a-a^{2} / 2$. This bound is equivalent to the "fourthegy bound" of Subramanian and Hajek [7]. Also note that the RHS of (20) is non-negative (divide through by $K$ and apply Jensen's inequality to the second logarithmic term).

Example 1 (IEEE UWB Model) The fading coefficients for the IEEE UWB channel model [4] are not complex Gaussian as mentioned above. We will nevertheless compute the bound (20) using the 2nd order statistics of the IEEE model to 
obtain an estimate of the achievable rates on the UWB channel. We fix the coherence time as $T_{c}=200 \mu \mathrm{s}$ and the $S N R$ as $\mathcal{E}_{s} / N_{0}=-3.88 \mathrm{~dB}$ corresponding to a range of $10 \mathrm{~m}$. For computations, we use the 100 CM/4 channel realizations as generated by the MATLAB code given in [4]. We consider systems with $R F$ bandwidths $W$ ranging from $500 \mathrm{MHz}$ to $6 \mathrm{GHz}$ in steps of $500 \mathrm{MHz}$. The-sampling rate equals $T_{s}=1 / \mathrm{W}$. A carrier frequency $f_{c}=5.092 \mathrm{GHz}$ is used in computing the vector $g$ as given by (3). We leave the mean channel energy $\overline{\|g\|^{2}}$ unnormalized; it equals 1.4277 when averaged over the 100 sample realizations. Fig. 1 shows the bound (20) after time-normalization through division by $T_{c}$.

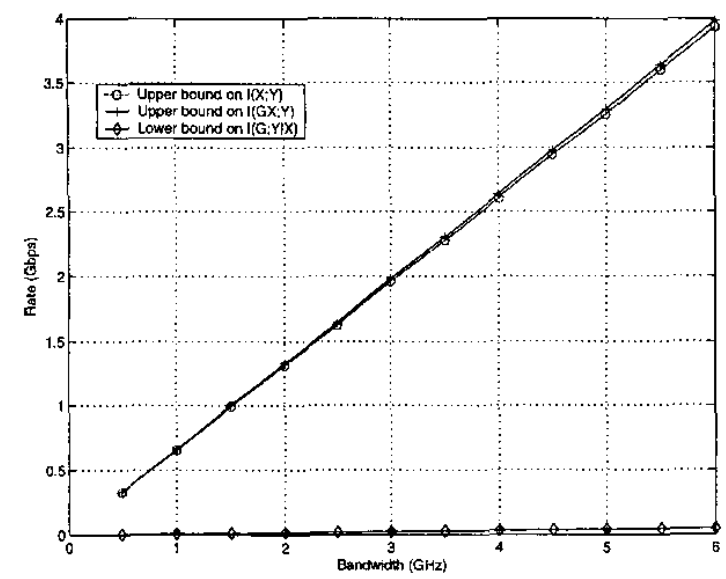

Figure 1: Upper bound of Proposition 2 on $I(X ; Y) / T_{c}$.

It may be of interest to note that for the same set of parameters the fourthegy bound (21) ranges from 293 to 3941 Gbps as $W$ ranges from $500 \mathrm{MHz}$ to $6 \mathrm{GHz}$. The fourthegy bound does not go to zero as $W$ is increased because the signal power scales linearly with $W$, unlike typical scenarios for wireless communications where the total transmitter power is fixed. In other words, for the UWB channel the SNR in each degree of freedom can be maintained at a constant level even as one uses more degress of freedom; this prevents the signal from being "overspread."

In fact, for the above set of parameters, the correction term $I(G ; Y \mid X)$ is negligible compared to $I(X G ; Y)$ which indicates that the coherence time is long enough to carry out sufficient amount of training at negligible cost to the overall rate. This picture changes, however, if one considers sending short packets over the UWB channel. Then, we need to replace $K$ by the number of samples in one packet duration. To study this case, consider packets of length $1 \mu \mathrm{s}$, which in effect is equivalent to setting $T_{c}=1 \mu \mathrm{s}$. (We are assuming that packets are sent intermittently with mean inter-transmission times greater than the actual channel coherence time of $200 \mu \mathrm{s}$. So, any channel state information gained in one packet's reception is useless for future packets.) Fig. 2 shows the bounds on the mutual information terms for this case. The bound on $I(G X ; Y) / T_{c}$ is the same as before. While before $I(G ; Y \mid X) / T_{\mathrm{c}}$ was negligible compared to $I(G X ; Y) / T_{c}$, now it is quite significant.

\section{A LOWER BOUND ON ACHIEVABle RATES}

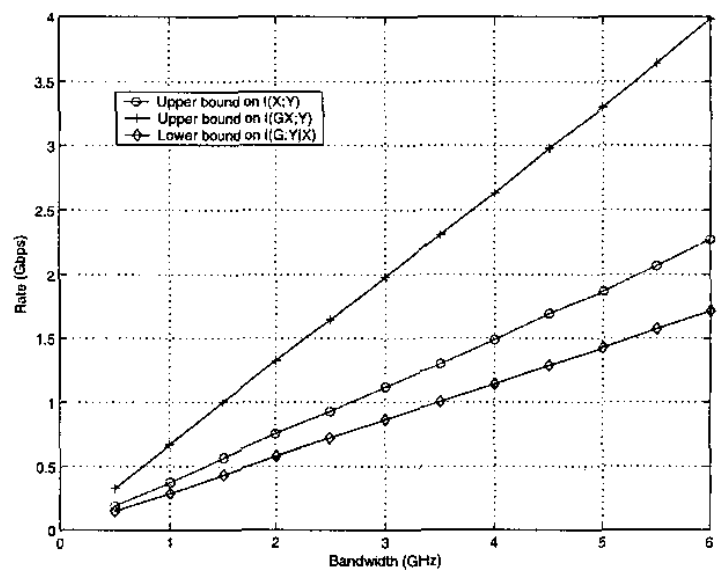

Figure 2: Upper bound of Proposition 2 on $I(X ; Y) / T_{c}$ for short packets.

To give a lower bound on $I(X ; Y)$, we follow the argument in [6] with some adjustment for the unequal path strengths. The lower bound is based on

$$
\begin{aligned}
I(X ; Y) & =I(X G ; Y)-I(G ; Y \mid X) \\
& \geq I(X ; Y \mid G)-I(G ; Y \mid X)
\end{aligned}
$$

We now take $X$ as complex Gaussian with $X \sim C N\left(0, \mathcal{E}_{s} I_{K}\right)$. Then,

$$
I(X ; Y \mid G)=E_{G}\left[\sum_{i=0}^{K-1} \log \left(1+\frac{K \mathcal{E}_{s}\left|G_{i}\right|^{2}}{N_{0}}\right)\right]
$$

This is true for any distribution on the path gains $g$. If we assume that $g$ has independent c.s. components, then the components of $G$ are identically-distributed, and we have

$$
I(X ; Y \mid G)=K E_{G_{0}}\left[\log \left(1+\frac{K \mathcal{E}_{s}\left|G_{0}\right|^{2}}{N_{0}}\right)\right]
$$

To upper-bound the term $I(G ; Y \mid X)$, consider the timedomain channel representation and let $s=g \circledast x$. The covariance of $s$ conditional on a given $x$ equals $B C_{g} B^{\dagger}$ where $B$ is the $K \times K$ matrix with elements $B_{i j}=x_{(i-j)}$. Using the Gaussian upper bound on mutual information for a given covariance,

$$
\begin{aligned}
I(G ; Y \mid X) & =E\left[\log \operatorname{det}\left(I_{K}+\frac{1}{N_{0}} B C_{g} B^{\dagger}\right)\right] \\
& =E\left[\log \operatorname{det}\left(I_{K}+\frac{1}{N_{0}} B^{\dagger} B C_{g}\right)\right] \\
& \leq E\left[\frac{1}{2} \log \prod_{j=0}^{K-1}\left\|A^{(j)}\right\|^{2}\right] \\
& \leq \sum_{j=0}^{K-1} \frac{1}{2} \log E\left[\left\|A^{(j)}\right\|^{2}\right]
\end{aligned}
$$

where the first inequality is Hadamard's inequality [9, p. 153] on the matrix $A \triangleq I+\frac{1}{N_{0}} B^{\dagger} B C_{g}$ and $A^{(j)}$ denotes the $j$ th 
column of $A$. The elements of $A$ are

$$
A_{i j}=\delta_{i j}+\frac{K \mathcal{E}_{s} \overline{\left|g_{j}\right|^{2}}}{N_{0}} \phi_{x}(i-j)
$$

where

$$
\phi_{x}(i-j)=\frac{1}{K \mathcal{E}_{s}} \sum_{k=0}^{K-1} x_{(k-i)}^{*} x_{(k-j)}
$$

is the normalized empirical autocorrelation of the sequence $x$. It can be computed that

$$
\begin{aligned}
E\left[\left\|A^{(j)}\right\|^{2}\right] & =\left(1+\frac{K \mathcal{E}_{s} \overline{\left|g_{j}\right|^{2}}}{N_{0}}\right)^{2}+\left(K^{2}+\nu K\right) \frac{\mathcal{E}_{s}^{2}\left[\overline{\left|g_{j}\right|^{2}}\right]^{2}}{N_{0}^{2}} \\
& \leq\left(1+\frac{2 K \mathcal{E}_{s} \overline{\left|g_{j}\right|^{2}}}{N_{0}}\right)^{2}
\end{aligned}
$$

where $\nu=1$ or 2 depending on $K$ being odd or even, respectively. This gives the bound

$$
I(G ; Y \mid X) \leq \sum_{i=0}^{K-1} \log \left(1+\frac{2 K \mathcal{E}_{s} \overline{\left.g_{i}\right|^{2}}}{N_{0}}\right)
$$

Thus, we have the following lower bound on channel capacity.

Proposition 3 The mutual information $I(X ; Y)$ over the channel (2), with $X \sim C N\left(0, \mathcal{E}_{s} I_{K}\right)$, is lower-bounded by

$$
E_{G}\left[\sum_{i=0}^{K-1} \log \left(1+\frac{K \mathcal{E}_{s}\left|G_{i}\right|^{2}}{N_{0}}\right)\right]-\sum_{i=0}^{K-1} \log \left(1+\frac{2 K \mathcal{E}_{s} \overline{\left|g_{i}\right|^{2}}}{N_{0}}\right)
$$

Example 2 (UWB capacity lower bound) We consider the same scenario as in Ex. 1 and compute the lower bound of Prop. 3. We have approximated the distribution of $\left|G_{i}\right|$ with that of $\left|G_{0}\right|$ in these computations. The resulting bounds are shown in Fig. 3 for $T_{c}=200 \mu$ s and in Fig. 4 for $T_{c}=1$ $\mu s$. More work needs to be done to close the gap between these bounds and those of Ex. 1 .

\section{CONCLUSIONS}

We have obtained upper and lower bounds on the achievable rate for the 802.15.3a UWB channel model under certain assumptions and approximations on the distributions of path gains $g$ and channel inputs $X$. The results show that the enormous bandwidth offered by the UWB channel can be utilized effectively by signaling schemes that spread the energy uniformly across each degree of freedom. This result is not surprising in view of the fact that in the UWB channel model considered here the signal power is allowed to grow with the degrees of freedom employed. If the total signal power is fixed, the results of [8] and [7] show that such uniform spreading of signal energy across the available degrees of freedom leads to a collapse of achievable rates beyond a certain point. Another conclusion is that under the target operating conditions for a UWB personal area network, it appears that relatively quick estimation of the channel state is possible, suggesting the use of feedback schemes for better channel utilization. We propose finding better methods for estimating the UWB channel capacity and devising practical signaling schemes that can efficiently utilize the channel capacity as subjects for further study.

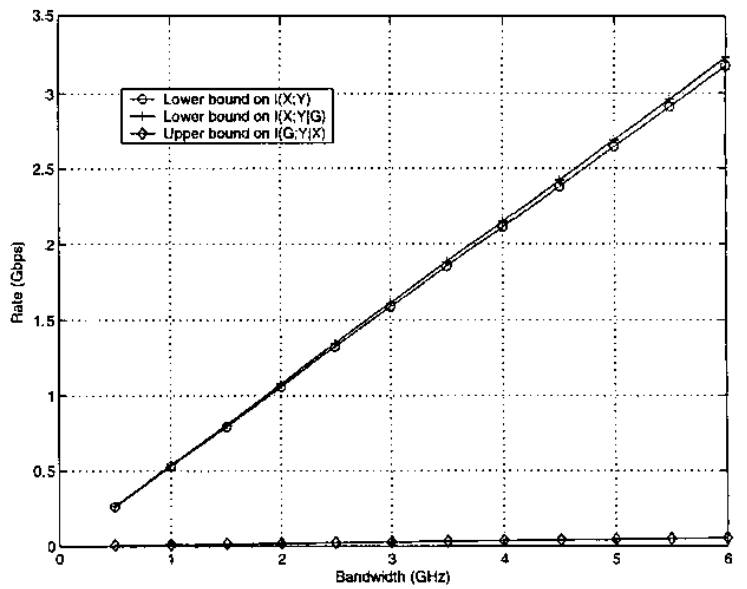

Figure 3: Lower bound of Proposition 3 on $I(X ; Y) / T_{c}$.

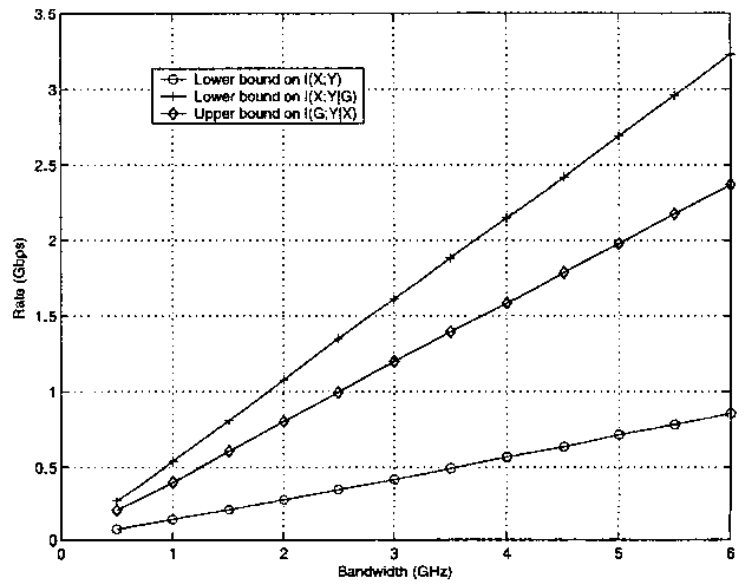

Figure 4: Lower bound of Proposition 3 on $I(X ; Y) / T_{c}$ for packet of length $1 \mu \mathrm{s}$.

\section{REFERENCES}

[1] "First Report and Order in the Matter of Revision of Part 15 of the Commission's Rules Regarding Ultra-Wideband Transmission Systems," FCC, ET Docket 98-153, FCC 02-48, Apr. 22, 2002.

[2] "UWB Channel Modeling Contribution from Intel," 24 June 2002, IEEE P802.15-02/279r0-SG3a. Online: http://grouper.ieee.org/groups/802/15/pub/2002/Jul02/

[3] "Multi-band OFDM Physical Layer Proposal for IEEE 802.15 Task Group 3a," 21 July 2003, IEEE P802.15-03/268r0. Online: http://grouper.ieee.org/groups/802/15/pub/2003/Ju103/

[4] "Channel Modeling Sub-committee Report," Dec. 2002, IEEE P802.15-02/368r5-SG3a. Online: http://grouper.ieee.org/groups/802/15/pub/2002/Nov02/

[5] W. Hirt and J. L. Massey, "Capacity of the discrete-time Gaussian channel with intersymbol interference" IEEE Trans. Info. Theory, vol. IT-34, pp. 380 - 388, May 1988.

[6] I. E. Telatar and D. Tse, "Capacity and mutual information 
of wideband multipath fading channels," IEEE Trans. Inform. Theory, vol. 46, no. 4, pp. 1384-1400, July 2000.

[7] V. G. Subramanian and B. Hajek, "Broad-band fading channels: signal burstiness and capacity," IEEE Trans. Inform. Theory, vol. 48, no. 4, pp. 809-827, April 2002.

[8] M. Médard and R. G. Gallager, "Bandwidth scaling for fading multipath channels," IEEE Trans. Inform. Theory, vol. 48, no. 4, pp. 840-852, April 2002.

[9] J. N. Franklin, Matrix Theory. Englewood Cliffs, New Jersey: Prentice-Hall, 1968. 